\title{
Associations between tree nut consumption and diet quality in the UK adult population based on National Diet and Nutrition Survey (NDNS) rolling programme 2008-2014
}

\author{
V. Dikariyanto ${ }^{1}$, W.L. Hall ${ }^{1}$, S.E.E. Berry ${ }^{1}$ and G.K. Pot ${ }^{1,2}$ \\ ${ }^{1}$ Diet and Cardiometabolic Disease Research Group, Faculty of Life Science and Medicine, King's College London, \\ London SE1 9NH, UK and \\ ${ }^{2}$ Vrije Universiteit Amsterdam, The Netherlands
}

Tree nut consumption has been reported to be associated with better diet quality according to evidence from the National Health and Nutrition Examination Survey (NHANES) in the US adult population ${ }^{(1)}$. Our study provides complementary information for the UK adult population using data from the UK National Diet and Nutrition Survey (NDNS) rolling programme (2008-2014) ${ }^{(2)}$. The aim was to investigate associations between tree nut consumption and overall diet quality in adults.

Cross sectional analysis was carried out using data from the NDNS rolling programme (2008-2014), including data from 3,371 individuals aged $\geq 19$ y who completed a 4-d estimated food diary. Tree nut consumption was defined as: 1) nut snacks only, 2) total consumption including composite dishes. To estimate diet quality, two different approaches were used: 1) a data-driven approach with principle component analysis (PCA) using 59 food items, 2) a hypothesis-driven approach with 3 existing diet quality scores (Mediterranean Diet Score (MDS; potential score range 0-9) ${ }^{(3)}$, modified Healthy Diet Score (modified HDS; potential score range $0-14)^{(4)}$ and Eating Choices Index (ECI; potential score range 4-20) $)^{(5)}$. Multivariate linear regression models were used to investigate associations between tree nut consumption and diet quality taking into account covariates for age, sex, ethnicity, socioeconomic status, smoking status, alcohol intake and energy intake.

In the data-driven approach, PCA identified 3 dietary patterns for tree nut snack consumers and 4 patterns for non-consumers, as well as 5 dietary patterns for tree nut consumption including composite dishes and 6 patterns for composite dish non-consumers. The table presents the associated food items included in the primary dietary patterns for tree nut snack consumption (Component 1 contributing the highest percentage of variation explained).

\begin{tabular}{lll}
\hline & Tree nut snack consumers, $\mathrm{n}=416$ & Tree nut snack non-consumers, $\mathrm{n}=2955$ \\
\hline Component 1 & + Fruit & + White bread \\
& + Other potatoes, potato salad and dishes & + Soft drinks not low calorie \\
& + Tea, coffee and water & - Fruit \\
+ Vegetables not raw & + Chips, fried and roast potatoes and potato products \\
& + Buns, cakes, pastries and fruit pies & + Crisps and savoury snacks \\
& + Yogurt, fromage frais and dairy dessert & - High fibre breakfast cereals \\
& + Salad and other raw vegetables & - Wholemeal bread \\
& + High fibre breakfast cereals & - Oily fish \\
& + Cheese & - Tea, coffee and water \\
& (4.3\% of variation explained) & + Burgers and kebabs \\
& + Coated, chicken and turkey \\
& - Yogurt, fromage frais and dairy dessert
\end{tabular}

Included items had factor loadings $>0 \cdot 3 .+$ shows a positive association, - shows a negative association.

For the hypothesis-driven approach, tree nut consumption, both as snack and in composite dishes, significantly predicted diet quality using all 3 scores: MDS, modified HDS and ECI $(p \leqslant 0.001)$. For every gram increase in tree nuts included in composite dish consumption per $1000 \mathrm{kcal}$ of adult's total energy intake, MDS significantly increased $0 \cdot 331$ ( $\mathrm{p}<0.001)$. However, tree nut snack consumption alone did not statistically change the diet quality scores for every gram of the consumption per $1000 \mathrm{kcal}$ of total energy intake. Consumption of tree nuts was associated with dietary patterns including lower processed foods and refined carbohydrates as well as higher fruit and dietary fibre intakes. Tree nut consumption including composite dishes was associated with better diet quality indicated by the MDS.

1. O'Neil C, Nicklas T, Fulgoni V (2015) Nutrients 7, 595-607.

2. National Diet and Nutrition Survey. (2008/2009-2013/14). Sep 2016. Public Health England/UK Food Standards Agency.

3. Trichopoulou A, Bamia C, Lagiou P et al. (2010) Am J Clin Nutr 92(3), 620-625.

4. Maynard M, Ness AR, Abraham L et al. (2005) Public Health Nutr 8, 321-326.

5. Pot GK, Richards M, Prynne CJ et al. (2014) Public Health Nutr 17(12), 2660-2666. 